Arch. Math. 95 (2010), 325-332

(C) 2010 The Author(s).

This article is published

with open access at Springerlink.com

0003-889X/10/040325-8

published online August 31, 2010

DOI 10.1007/s00013-010-0172-7

Archiv der Mathematik

\title{
A note on termination of the Baer construction of the prime radical
}

\author{
M. A. Chebotar, P.-H. Lee, and E. R. Puczyłowski
}

\begin{abstract}
The well known Baer construction of the prime radical shows that the prime radical of an arbitrary ring is the union of the chain of ideals of the ring, constructed by transfinite induction, which starts with 0 and repeats the procedure of taking the sum of ideals that are nilpotent modulo ideals in the chain already constructed. Amitsur showed that for every ordinal number $\alpha$ there is a ring for which the construction stops precisely at $\alpha$. In this paper we construct such examples with some extra properties. This allows us to construct, for every countable non-limit ordinal number $\alpha$, an affine algebra for which the construction terminates precisely at $\alpha$. Such an example was known due to Bergman for $\alpha=2$.
\end{abstract}

Mathematics Subject Classification (2000). 16A21, 16A22.

Keywords. Prime radical, Baer chain, Affine algebra.

In this paper we consider associative algebras over a fixed field $F$. In what follows the term "algebra" will mean $F$-algebra not necessarily with unity. For a given algebra $A$ without unity, we denote by $A^{*}$ the algebra obtained from $A$ by the adjunction of a unity. For an algebra $A$ with unity, we set $A^{*}$ to be $A$ itself.

To denote that $I$ is a two-sided ideal (left ideal) of an algebra $A$ we write $I \triangleleft A$ (respectively, $I<_{l} A$ ). Obviously if $L$ is a left ideal of $A$, then the two-sided ideal of $A$ generated by $L$ is equal to $L A^{*}$.

Denote for a given algebra $A$ by $W(A)$ the sum of all nilpotent ideals of $A$. Since a left (right) ideal of $A$ is nilpotent if and only the two-sided ideal it generates is nilpotent, $W(A)$ is also the sum of all nilpotent left (right) ideals

E. R. Puczyłowski was supported by MNiSW Grant Nr N N201 268435 and National Center for Theoretical Sciences, Taipei Office, Taiwan. 
of $A$. Moreover, it is clear that $a \in W(A)$ if and only if the principal left ideal $A^{*} a$ of $A$ is nilpotent.

Define inductively for ordinal numbers $\alpha$ the ideals $W_{\alpha}(A)$ as follows.

- $W_{0}(A)=0$.

- Suppose that $\alpha>0$ and $W_{\beta}(A)$ are defined for all $\beta<\alpha$.

(a) If $\alpha$ is a limit ordinal number, then $W_{\alpha}(A)=\bigcup_{\beta<\alpha} W_{\beta}(A)$.

(b) If $\alpha$ is not a limit ordinal number, then $W_{\alpha}(A)$ is the ideal of $A$ containing $W_{\alpha-1}(A)$ such that $W_{\alpha}(A) / W_{\alpha-1}(A)=W\left(A / W_{\alpha-1}(A)\right)$, or equivalently $W_{\alpha}(A)$ is the sum of all ideals (left ideals) $I$ of $A$ such that $I$ is nilpotent modulo $W_{\alpha-1}(A)$.

Thus, for a non-limit ordinal number $\alpha$, we have $a \in W_{\alpha}(A)$ if and only if the principal left ideal $A^{*} a$ of $A$ is nilpotent modulo $W_{\alpha-1}(A)$.

It is well known that the above defined chain of ideals of $A$ stabilizes at the prime radical $\mathcal{P}(A)$ of $A$. It is called the Baer construction of the prime radical.

In [1] Amitsur constructed examples showing that for every ordinal number $\alpha>0$ there is an algebra $A$ such that $A=W_{\alpha}(A) \neq W_{\beta}(A)$ for every $\beta<\alpha$. In such a situation we say that the Baer chain of $A$ terminates at $\alpha$. There is also an example due to Bergman [4] of an affine algebra of which the Baer chain terminates at 2 .

In this note we construct another, and simpler as we believe, example giving Amitsur's result that satisfies some extra conditions. Applying it we extend Bergman's result by constructing for every countable non-limit (in particular finite) ordinal number $\alpha$ an affine algebra $A$ such that the Baer chain of $A$ terminates at $\alpha$.

We start with collecting some basic properties of $W_{\alpha}(A)$ which will be needed later. Some of them can be easily verified by applying straightforward induction arguments. For instance, $f\left(W_{\alpha}(A)\right) \subseteq W_{\alpha}(f(A))$ for any algebra homomorphism $f: A \rightarrow A^{\prime}$. Moreover, if $I \triangleleft A$ is nilpotent, then $I \subseteq W_{\alpha}(A)$ and $W_{\alpha}(A / I)=W_{\alpha}(A) / I$ for any $\alpha>0$. More properties of $W_{\alpha}(A)$ are listed in the following propositions.

Proposition 1. Let $A$ be an algebra and $\alpha$ an arbitrary ordinal number.

(1) If $L<_{l} A$, then $W_{\alpha}(A) \cap L \subseteq W_{\alpha}(L)$.

(2) If $I \triangleleft A$, then $W_{\alpha}(I)=I \cap W_{\alpha}(A)$ and so $W_{\alpha}(I) \triangleleft A$ and $W_{\alpha}(I) \subseteq W_{\alpha}(A)$.

(3) If $L<_{l} A$, then $L W_{\alpha}(L) \subseteq W_{\alpha}(A)$.

(4) If $L<_{l} A$ and $W_{\alpha}(L)=L$, then $\left(L A^{*}\right)^{2} \subseteq W_{\alpha}\left(L A^{*}\right)$ and so $W_{\alpha+1}\left(L A^{*}\right)$ $=L A^{*}$.

Proof. Note first that (4) is a consequence of (2) and (3). Indeed, if $W_{\alpha}(L)=$ $L<_{l} A$, then $L<_{l} L A^{*} \triangleleft A$ and $L^{2}=L W_{\alpha}(L) \subseteq W_{\alpha}\left(L A^{*}\right)$ by (3). Since $W_{\alpha}\left(L A^{*}\right) \triangleleft A$ by $(2)$, we have $\left(L A^{*}\right)^{2} \subseteq L^{2} A^{*} \subseteq W_{\alpha}\left(L A^{*}\right)$, which obviously implies that $W_{\alpha+1}\left(L A^{*}\right)=L A^{*}$.

To prove (1)-(3), we proceed by induction on $\alpha$. All of these are clear for $\alpha=0$. Thus assume that $\alpha>0$ and they are satisfied for ordinal numbers smaller than $\alpha$. Clearly they are also satisfied if $\alpha$ is a limit ordinal number, so assume that it is non-limit. 
(1) Take any $l \in L \cap W_{\alpha}(A)$. Then the left ideal $A^{*} l$ of $A$ is nilpotent modulo $W_{\alpha-1}(A)$. Since $W_{\alpha-1}(A) \cap L \subseteq W_{\alpha-1}(L)$ by the induction assumption, the left ideal $A^{*} l$ of $L$ is also nilpotent modulo $W_{\alpha-1}(L)$. Consequently $l \in W_{\alpha}(L)$ and we are done.

(2) In view of (1) it suffices to show only that $W_{\alpha}(I) \subseteq W_{\alpha}(A)$. Take any ideal $K$ of $I$ such that $K^{n} \subseteq W_{\alpha-1}(I)$ for some $n$. Set $\bar{K}=A^{*} K A^{*} \triangleleft A$. Then $\bar{K}^{3} \subseteq I K I \subseteq K$ and so $\bar{K}^{3 n} \subseteq W_{\alpha-1}(I) \subseteq W_{\alpha-1}(A)$ by the induction assumption. Hence $K \subseteq \bar{K} \subseteq W_{\alpha}(\bar{A})$. Consequently the inclusion $W_{\alpha}(I) \subseteq W_{\alpha}(A)$ holds.

(3) Let $K$ be an ideal of $L$ such that $K^{n} \subseteq W_{\alpha-1}(L)$ for some $n$. Then $L K<_{l} A$ and $(L K)^{n} \subseteq L K^{n} \subseteq L W_{\alpha-1}(L) \subseteq W_{\alpha-1}(A)$ by the induction assumption. Consequently $L K \subseteq W_{\alpha}(A)$. This obviously implies that $L W_{\alpha}(L) \subseteq W_{\alpha}(A)$.

Proposition 2. If $A=\oplus_{i \in I} A_{i}$, then $W_{\alpha}(A)=\oplus_{i \in I} W_{\alpha}\left(A_{i}\right)$ for every $\alpha$.

Proof. Since $A_{i} \triangleleft A$ for each $i \in I$, we have that $W_{\alpha}\left(A_{i}\right) \subseteq W_{\alpha}(A)$ by Proposition 1 (2) and so $\oplus_{i \in I} W_{\alpha}\left(A_{i}\right) \subseteq W_{\alpha}(A)$ for every $\alpha$. Note that $A$ is an ideal of $\oplus_{i \in I} A_{i}^{*}$. Hence $W_{\alpha}(A)$ is also an ideal of $\oplus_{i \in I} A_{i}^{*}$ by Proposition 1 (2). This implies that $W_{\alpha}(A)=\oplus_{i \in I} J_{i}$ for some $J_{i} \triangleleft A_{i}$. Obviously each canonical projection of $A$ onto $A_{i}$ maps $W_{\alpha}(A)$ onto $J_{i}$. Hence $J_{i} \subseteq W_{\alpha}\left(A_{i}\right)$ and so $W_{\alpha}(A) \subseteq \oplus_{i \in I} W_{\alpha}\left(A_{i}\right)$. Thus the result follows.

In our construction we shall employ some infinite matrix algebras.

For a given algebra $A$, we denote by $M(A)$ the algebra of all columnfinite matrices indexed by positive integers with entries in $A$, and by $M^{f}(A)$ the subalgebra of $M(A)$ consisting of all matrices with finitely many nonzero entries. Note that $M^{f}(A)<_{l} M(A)$. Obviously $M^{f}(A) / M^{f}(I) \simeq M^{f}(A / I)$ and $M(A) / M(I) \simeq M(A / I)$ for any ideal $I$ of $A$.

For given positive integers $i, j$ and given $m \in M(A)$ we denote by $m_{i j}$ the $(i, j)$-entry of $m$ and by $e_{i j}$ the matrix in $M^{f}\left(A^{*}\right)$ with 1 as its $(i, j)$-entry and zeros elsewhere.

Note that if $A$ is an algebra without unity, then $M(A) \triangleleft M\left(A^{*}\right), M(F)$ is a subalgebra of $M\left(A^{*}\right), M\left(A^{*}\right)=M(A)+M(F)$ and $M(A) \cap M(F)=0$.

We shall need the following lemma proved in [2]. We duplicate its short proof here for completeness.

Lemma 3. Let $A$ be a Jacobson radical algebra and $L$ a left $M^{f}(A)$-submodule of $M\left(A^{*}\right)$ such that $L \nsubseteq M(A)$. If $L$ is nilpotent, so is $A$.

Proof. Since $L \nsubseteq M(A)$, there is a matrix $m \in L$ such that $m_{s t} \in A^{*} \backslash A$ for some positive integers $s, t$. Now $A$ is Jacobson radical, so there is $b \in A^{*}$ such that $b m_{s t}=m_{s t} b=1$. Then $A b e_{t s} m=\left\{\sum_{j} a b m_{s j} e_{t j} \mid a \in A\right\} \subseteq L$. Note that $e_{t s} m A b e_{t s} \subseteq A e_{t s}$ and $b A=A b=A$, so $A b e_{t s} m$ is a subalgebra of $M(A)$ contained in $L$. Moreover the map $a b e_{t s} m \rightarrow a b e_{t s} m e_{t t}=a b m_{s t} e_{t t}=a e_{t t}$ is an epimorphism of $A b e_{s t} m$ onto $A e_{t t} \simeq A$. These show that $A$ is nilpotent if $L$ is nilpotent.

We shall also need the following lemma. 
Lemma 4. Let $T$ be a subalgebra of $M(A)$ containing $M^{f}(A)$. Then $M^{f}$ $\left(W_{\alpha}(A)\right) \subseteq W_{\alpha}(T) \subseteq M\left(W_{\alpha}(A)\right)$ for every ordinal number $\alpha$.

Proof. We proceed by induction on $\alpha$. For $\alpha=0$ the result is obvious. Thus assume that $\alpha>0$ and it holds for ordinal numbers smaller than $\alpha$. It also holds if $\alpha$ is a limit ordinal number, so assume that $\alpha$ is non-limit. Note that $M^{f}\left(W_{\alpha}(A)\right)$ is the sum of ideals $M^{f}(I)$ where $I$ is an ideal of $A$ which is nilpotent modulo $W_{\alpha-1}(A)$. Now each such $M^{f}(I)=\sum_{n} M^{f}(I) e_{n n}$ and $M^{f}(I) e_{n n}$ is a left ideal of $T$ which is nilpotent modulo $M^{f}\left(W_{\alpha-1}(A)\right) \subseteq W_{\alpha-1}$ $(T)$ by the induction assumption. Consequently $M^{f}\left(W_{\alpha}(A)\right) \subseteq W_{\alpha}(T)$.

Take any ideal $I$ of $T$ which is nilpotent modulo $W_{\alpha-1}(T)$ and set $I_{i j}=$ $\left\{m_{i j} \mid m \in I\right\}$ for arbitrary positive integers $i, j$. Then $I_{i j} e_{i i}=e_{i i} I e_{j i} \triangleleft$ $e_{i i} T e_{j i}=A e_{i i}$ since $M^{f}(A) \subseteq T$. Thus $I_{i j} e_{i i}=e_{i i} I e_{j i}$ is nilpotent modulo $e_{i i} W_{\alpha-1}(T) e_{j i}=W_{\alpha-1}(A) e_{i i}$ since $M^{f}\left(W_{\alpha-1}(A)\right) \subseteq W_{\alpha-1}(T) \subseteq M\left(W_{\alpha-1}\right.$ $(A)$ ) by the induction assumption. Hence $I_{i j}$ is nilpotent modulo $W_{\alpha-1}(A)$ and so $I_{i j} \subseteq W_{\alpha}(A)$ for arbitrary positive integers $i, j$. Consequently $W_{\alpha}(T) \subseteq$ $M\left(W_{\alpha}(A)\right)$ and we are done.

Now we are ready to prove our first main result. For a given ordinal number $\alpha$ we denote by $\operatorname{card}(\alpha)$ the cardinality of the set of all ordinal numbers smaller than $\alpha$.

Theorem 5. Let $S$ be a non-nilpotent algebra such that $\operatorname{dim}_{F} S=\aleph_{0}$ and $W(S)=S$. Then, for every ordinal number $\alpha$ there exists an algebra $A_{\alpha}$ such that

(a) $\operatorname{dim}_{F} A_{\alpha} \leq \max \left\{\aleph_{0}, \operatorname{card}(\alpha)\right\}$;

(b) $W_{\alpha}\left(A_{\alpha}\right)=A_{\alpha}$ and $A_{\alpha} / W_{\beta}\left(A_{\alpha}\right)$ is not nilpotent for $\beta<\alpha$;

(c) $A_{\alpha} / W_{\alpha-1}\left(A_{\alpha}\right) \simeq S$ if $\alpha$ is non-limit.

Proof. Applying the regular representation of $S^{*}$ we get an embedding of $S$ into $\operatorname{End}_{F}\left(S^{*}\right) \simeq M(F)$, so we may assume that $S \subseteq M(F)$.

We proceed by induction on $\alpha$. Obviously $A_{0}=0$ and $A_{1}=S$ satisfy the requirements for $\alpha=0,1$. Thus assume that $\alpha>1$ and we have already constructed $A_{\gamma}$ for $\gamma<\alpha$. If $\alpha$ is a limit ordinal, we take $A_{\alpha}=\oplus_{\gamma<\alpha} A_{\gamma}$. Then $\operatorname{dim}{ }_{F} A_{\alpha} \leq \operatorname{card}(\alpha) \max \left\{\aleph_{0}, \operatorname{card}(\alpha)\right\}=\max \left\{\aleph_{0}, \operatorname{card}(\alpha)\right\}$. In view of Proposition 2, we have $W_{\alpha}\left(A_{\alpha}\right)=\oplus_{\gamma<\alpha} W_{\alpha}\left(A_{\gamma}\right)=\oplus_{\gamma<\alpha} A_{\gamma}=A_{\alpha}$ and, for $\beta<\alpha, W_{\beta}\left(A_{\alpha}\right)=\oplus_{\gamma<\alpha} W_{\beta}\left(A_{\gamma}\right)=\left(\oplus_{\gamma \leq \beta} A_{\gamma}\right) \oplus\left(\oplus_{\beta<\gamma<\alpha} W_{\beta}\left(A_{\gamma}\right)\right)$, and so $A_{\alpha} / W_{\beta}\left(A_{\alpha}\right) \simeq \oplus_{\beta<\gamma<\alpha} A_{\gamma} / W_{\beta}\left(A_{\gamma}\right)$. Note that $A_{\beta+1} / W_{\beta}\left(A_{\beta+1}\right) \simeq S$ is not nilpotent by the induction assumption. Hence $A_{\alpha} / W_{\beta}\left(A_{\alpha}\right)$ is not nilpotent either.

Thus suppose that $\alpha$ is a non-limit ordinal number. Let $A_{\alpha}$ be the subalgebra of $M\left(A_{\alpha-1}{ }^{*}\right)$ generated by $M^{f}\left(A_{\alpha-1}\right)$ and $S$. Since $M^{f}\left(A_{\alpha-1}\right)<_{l}$ $M\left(A_{\alpha-1}{ }^{*}\right)$, we have $A_{\alpha}=T+S$ where $T=M^{f}\left(A_{\alpha-1}\right)+M^{f}\left(A_{\alpha-1}\right) S \triangleleft$ $A_{\alpha}$. Now $\operatorname{dim}_{F} S=\aleph_{0}$ and $\operatorname{dim}_{F} M^{f}\left(A_{\alpha-1}\right)=\aleph_{0} \operatorname{dim}{ }_{F} A_{\alpha-1} \leq \aleph_{0} \max \left\{\aleph_{0}\right.$, $\operatorname{card}(\alpha-1)\}=\max \left\{\aleph_{0}, \operatorname{card}(\alpha)\right\}$, so $\operatorname{dim}_{F} A_{\alpha} \leq \max \left\{\aleph_{0}, \operatorname{card}(\alpha)\right\}+\aleph_{0} \max$ $\left\{\aleph_{0}, \operatorname{card}(\alpha)\right\}+\aleph_{0}=\max \left\{\aleph_{0}, \operatorname{card}(\alpha)\right\}$. Moreover, $M^{f}\left(A_{\alpha-1}\right)=M^{f}\left(W_{\alpha-1}\right.$ 
$\left.\left(A_{\alpha-1}\right)\right) \subseteq W_{\alpha-1}(T)$ by Lemma 4 and $W_{\alpha-1}(T) \subseteq W_{\alpha-1}\left(A_{\alpha}\right)$ by Proposition 1 (2). Hence $M^{f}\left(A_{\alpha-1}\right) \subseteq W_{\alpha-1}\left(A_{\alpha}\right)$. Now $W_{\alpha-1}\left(A_{\alpha}\right) \triangleleft A_{\alpha}$, so $T=$ $M^{f}\left(A_{\alpha-1}\right)+M^{f}\left(A_{\alpha-1}\right) S \subseteq W_{\alpha-1}\left(A_{\alpha}\right)$.

In fact, we have $W_{\alpha-1}\left(A_{\alpha}\right)=T$. Assume on the contrary that $W_{\alpha-1}\left(A_{\alpha}\right) \not$ $T$ and let $\beta$ be the smallest ordinal number such that $W_{\beta}\left(A_{\alpha}\right) \nsubseteq T$. Clearly $\beta$ is not a limit ordinal number. Note that $W_{\beta-1}\left(A_{\alpha-1}\right) \triangleleft A_{\alpha-1}{ }^{*}$ by Proposition 1 (2). Thus $I=M\left(W_{\beta-1}\left(A_{\alpha-1}\right)\right) \triangleleft M\left(A_{\alpha-1}{ }^{*}\right)$ and $M\left(A_{\alpha-1}{ }^{*}\right) / I$ is canonically isomorphic to $M\left(\left(A_{\alpha-1} / W_{\beta-1}\left(A_{\alpha-1}\right)\right)^{*}\right)=M\left(A_{\alpha-1} / W_{\beta-1}\left(A_{\alpha-1}\right)\right)+M(F)$. Consider the canonical epimorphism $M\left(A_{\alpha-1}{ }^{*}\right) \rightarrow M\left(A_{\alpha-1} / W_{\beta-1}\left(A_{\alpha-1}\right)\right)+$ $M(F)$. Let $\bar{T}$ be the image of $T$ in $M\left(A_{\alpha-1} / W_{\beta-1}\left(A_{\alpha-1}\right)\right)$. Then $\bar{T}$ contains $M^{f}\left(A_{\alpha-1} / W_{\beta-1}\left(A_{\alpha-1}\right)\right)$ and so we get that $M^{f}\left(W\left(A_{\alpha-1} / W_{\beta-1}\left(A_{\alpha-1}\right)\right)\right) \subseteq$ $W(\bar{T})$ by Lemma 4 . Since $\bar{T} \triangleleft \bar{T}+S$, we have $W(\bar{T}) \subseteq W(\bar{T}+S)$ by Proposition $1(2)$ and so $M^{f}\left(W\left(A_{\alpha-1} / W_{\beta-1}\left(A_{\alpha-1}\right)\right)\right) \subseteq W(\overline{\bar{T}}+S)$. Now $W_{\beta-1}\left(A_{\alpha}\right) \subseteq$ $T \triangleleft A_{\alpha}$, so it follows from Lemma 4 and Proposition 1 (2) that $W_{\beta-1}\left(A_{\alpha}\right)=$ $W_{\beta-1}(T) \subseteq I$. Then $W_{\beta}\left(A_{\alpha}\right)$ is a sum of ideals of $A_{\alpha}$ which are nilpotent modulo $I$ and so $\left(W_{\beta}\left(A_{\alpha}\right)+I\right) / I \subseteq W\left(\left(A_{\alpha}+I\right) / I\right)$. On the other hand, $\left(W_{\beta}\left(A_{\alpha}\right)+I\right) / I \nsubseteq(T+I) / I$, for otherwise we would have $W_{\beta}\left(A_{\alpha}\right) \subseteq$ $T+I \subseteq M\left(A_{\alpha-1}\right)$ and so $W_{\beta}\left(A_{\alpha}\right) \subseteq A_{\alpha} \cap M\left(A_{\alpha-1}\right)=T$, a contradiction. Hence $W\left(\left(A_{\alpha}+I\right) / I\right) \nsubseteq(T+I) / I$. By the canonical isomorphism between $M\left(A_{\alpha-1}{ }^{*}\right) / I$ and $M\left(A_{\alpha-1} / W_{\beta-1}\left(A_{\alpha-1}\right)\right)+M(F)$, we have that $W(\bar{T}+S) \nsubseteq$ $\bar{T}$. Thus there exists a nilpotent left ideal $L$ of $\bar{T}+S$ that is not contained in $\bar{T}$ and so $L \nsubseteq M\left(A_{\alpha-1} / W_{\beta-1}\left(A_{\alpha-1}\right)\right)$. Then it follows from Lemma 3 that $A_{\alpha-1} / W_{\beta-1}\left(A_{\alpha-1}\right)$ is nilpotent, contradicting (b) since $\beta-1<\alpha-1$. Therefore $W_{\alpha-1}\left(A_{\alpha}\right)=T$ and so $A_{\alpha} / W_{\alpha-1}\left(A_{\alpha}\right)=A_{\alpha} / T \simeq S=W(S)$. Consequently $W_{\alpha}\left(A_{\alpha}\right)=A_{\alpha}$ and $A_{\alpha} / W_{\beta}\left(A_{\alpha}\right)$ is not nilpotent for any $\beta<\alpha$.

In particular, when $\alpha$ is finite or countably infinite, we have

Corollary 6. Let $S$ be a non-nilpotent algebra such that $\operatorname{dim}{ }_{F} S=\aleph_{0}$ and $W(S)=S$. Then, for every countable ordinal number $\alpha>0$ there exists an algebra $A_{\alpha}$ such that

$\left(a^{\prime}\right) \operatorname{dim}_{F} A_{\alpha}=\aleph_{0}$;

(b) $W_{\alpha}\left(A_{\alpha}\right)=A_{\alpha}$ and $A_{\alpha} / W_{\beta}\left(A_{\alpha}\right)$ is not nilpotent for $\beta<\alpha$;

(c) $A_{\alpha} / W_{\alpha-1}\left(A_{\alpha}\right) \simeq S$ if $\alpha$ is non-limit.

Proof. Let $A_{\alpha}$ be an algebra satisfying the conditions (a), (b), and (c) in Theorem 5 . In view of the proof of Theorem 5, we may assume further that for each $\gamma<\alpha A_{\alpha}$ contains a subalgebra $A_{\gamma}$ satisfying the conditions (a), (b), and (c) for $\gamma$ in place of $\alpha$. Since $\alpha$ is countable, we have $\operatorname{dim}_{F} A_{\alpha} \leq \aleph_{0}$ by (a). On the other hand, since $\alpha>0$, some subalgebra $A_{\gamma}$ of $A_{\alpha}$ can be mapped homomorphically onto $S$ by (c). Hence $\operatorname{dim}_{F} A_{\alpha} \geq \aleph_{0}$ and so $\left(\mathrm{a}^{\prime}\right) \operatorname{dim}{ }_{F} A_{\alpha}=\aleph_{0}$ holds.

It is not hard to find countable-dimensional non-nilpotent algebras $S$ such that $W(S)=S$. Among these the simplest examples is $S=\oplus_{n \geq 2}\left(x F[x] / x^{n}\right.$ $F[x]$ ), where $F[x]$ is the algebra of polynomials in indeterminate $x$ over $F$. Another example of such an algebra is the following Zassenhaus algebra. 
Let $S$ be the linear space over $F$ with the basis $x_{q}$, where $q$ are rational numbers larger than 0 and smaller than 1 . Define $x_{p} x_{q}=x_{p+q}$, when $p+q<1$ and 0 otherwise. One easily checks that $S$ is an idempotent algebra and $W(S)=S$.

Note that, choosing specific $S$ in the above theorem, we can get that the resulted algebras $A_{\alpha}$ have some additional properties. For instance, if we take $S$ to be idempotent, then all of these $A_{\alpha}$ are idempotent.

We conclude this note by applying Markov's idea [3] to obtain our second result.

Theorem 7. For every countable ordinal number $\alpha>0$ there exists an algebra $A_{\alpha}$ with unity generated by two elements such that $\mathcal{P}\left(A_{\alpha}\right)=W_{\alpha+1}\left(A_{\alpha}\right) \neq$ $W_{\alpha}\left(A_{\alpha}\right)$.

Proof. Let $R=F\langle x, y\rangle$ be the algebra of polynomials over $F$ in non-commuting indeterminates $x, y$. Then $L=R x<_{l} R, L R=R x R \triangleleft R$ and $R / L R \simeq F[y]$. Moreover, $L$ is a free $F$-algebra (without unity) with the set of free generators $x, y x, y^{2} x, \ldots$ By Corollary 6 there is an $F$-algebra $A$ such that $\operatorname{dim}_{F} A=\aleph_{0}$, $A=W_{\alpha}(A)$ and $A / W_{\gamma}(A)$ is not nilpotent for any $\gamma<\alpha$. Hence $L$ can be mapped homomorphically onto $A$, that is, $L / I \simeq A$ for some $I \triangleleft L$. Let $J=L I R \triangleleft R$. Then $I^{2} \subseteq J \cap L$ and $J \cap L=L I(R x+R y+F) \cap R x=$ $L I R x+L I=L I L+L I \subseteq I$, so the canonical isomorphism of $(L+J) / J$ onto $L /(J \cap L)$ maps $(I+J) / J$ onto $I /(J \cap L)$. Consequently, setting $\bar{L}=(L+J) / J$ and $\bar{I}=(I+J) / J$, we get that $\bar{L} / \bar{I} \simeq L / I \simeq A$. It is clear that $\bar{R}=R / J$ is an $F$-algebra with unity generated by two elements $\bar{x}, \bar{y}$, the images of $x, y$ in $\bar{R}$. We claim that $\bar{R}$ is a candidate for the required $A_{\alpha}$. Since $\bar{I}^{2}=0$ and $\bar{L} / \bar{I} \simeq A=W_{\alpha}(A)$, we have $W_{\alpha}(\bar{L}) / \bar{I}=W_{\alpha}(\bar{L} / \bar{I})=\bar{L} / \bar{I}$ and so $W_{\alpha}(\bar{L})=\bar{L}$. Hence $W_{\alpha+1}(\bar{L} \bar{R})=\bar{L} \bar{R}$ by Proposition 1 (4) and so $\bar{L} \bar{R} \subseteq W_{\alpha+1}(\bar{R}) \subseteq \mathcal{P}(\bar{R})$ by Proposition 1 (2). On the other hand, $\bar{R} / \bar{L} \bar{R} \simeq R / L R \simeq F[y]$ contains no nonzero nilpotent elements, so $\bar{L} \bar{R}=\mathcal{P}(\bar{R})=W_{\alpha+1}(\bar{R})$. Let $\beta$ be the smallest ordinal number such that $W_{\beta}(\bar{R})=\mathcal{P}(\bar{R})=\bar{R} \bar{x} \bar{R}$; then $\beta \leq \alpha+1$. Now $\bar{x}$ is not contained in any $W_{\gamma}(\bar{R})$ for $\gamma<\beta$, so $\beta$ is not a limit ordinal. Since $\bar{x} \in W_{\beta}(\bar{R}), \bar{L}=\bar{R} \bar{x}$ is nilpotent modulo $W_{\beta-1}(\bar{R}) \cap \bar{L} \subseteq W_{\beta-1}(\bar{L})$ by Proposition 1 (1). If $\beta=1$, then $\bar{L}$ would be nilpotent, contradicting that $\bar{L} / \bar{I} \simeq A$ is not nilpotent. Hence $\beta>1$. Note that the isomorphism of $A$ onto $\bar{L} / \bar{I}$ maps $W_{\beta-1}(A)$ onto $W_{\beta-1}(\bar{L} / \bar{I})=W_{\beta-1}(\bar{L}) / \bar{I}$, since $\bar{I}^{2}=0$. Thus $A / W_{\beta-1}(A) \simeq(\bar{L} / \bar{I}) /\left(W_{\beta-1}(\bar{L}) / \bar{I}\right) \simeq \bar{L} / W_{\beta-1}(\bar{L})$ is nilpotent. Hence $\beta-1 \geq$ $\alpha$ and so $\beta=\alpha+1$. Consequently $\mathcal{P}(\bar{R})=W_{\alpha+1}(\bar{R}) \neq W_{\alpha}(\bar{R})$.

From Theorem 7 it follows that for every countable non-limit ordinal number $\beta \geq 2$ there exists a finitely generated algebra of which the Baer chain terminates at $\beta$. The same obviously holds for $\beta=0$ or 1 . (One can take for $\beta=0$ any finitely generated semiprime algebra and for $\beta=1$ any finite-dimensional non-semiprime algebra.) However, we do not know whether the same is true also for countable limit ordinal numbers.

The algebra $A_{\alpha}$ that we constructed in the proof of Theorem 7 satisfies the property $\left(W_{\alpha+1}\left(A_{\alpha}\right) / W_{\alpha}\left(A_{\alpha}\right)\right)^{2}=0$ by Proposition 1 (4). One might ask whether it is possible to construct the desired algebra $A_{\alpha}$ so that $W_{\alpha+1}\left(A_{\alpha}\right) /$ $W_{\alpha}\left(A_{\alpha}\right)$ is not nilpotent (as we have seen for infinitely generated algebras it 
can be even idempotent). The answer for $\alpha=0$ is affirmative. As shown in [4, Lemma 8.1.16] there exists a finitely generated group $G$ such that the group algebra $A=K[G]$ of $G$ over any field $K$ of nonzero characteristic has a nonnilpotent $W(A)$ and by [4, Lemma 8.4.13] $\mathcal{P}(A)=W(A)$ for such an algebra $A$. We do not know the answer for $\alpha>0$.

Thus we leave the following questions open.

Question 8. Does there exist, for every countable limit ordinal number $\alpha, a$ finitely generated algebra of which the Baer chain terminates at $\alpha$ ?

Question 9. Does there exist, for every countable ordinal number $\alpha$, a finitely generated algebra $A_{\alpha}$ such that $\mathcal{P}\left(A_{\alpha}\right)=W_{\alpha+1}\left(A_{\alpha}\right) \neq W_{\alpha}\left(A_{\alpha}\right)$ and $W_{\alpha+1}$ $\left(A_{\alpha}\right) / W_{\alpha}\left(A_{\alpha}\right)$ is not nilpotent?

Open Access. This article is distributed under the terms of the Creative Commons Attribution Noncommercial License which permits any noncommercial use, distribution, and reproduction in any medium, provided the original author(s) and source are credited.

\section{References}

[1] S. A. Amitsur, Nil radicals, historical notes and some new results, Rings, Modules and Radicals (Proc. Internat. Colloq., Keszthely, 1971), 47-65. Colloq. Math. Soc. Janos Bolyai, 6, North-Holland, Amsterdam, 1973.

[2] M. Chebotar, P.-H. Lee, And E. R. PuczyŁowski, On Andrunakievich chain and Koethe's problem, Israel J. Math., to appear.

[3] V. T. Markov, Some examples of finitely generated algebras, (Russian) Uspekhi Mat. Nauk 36 (1981), 185-186.

[4] D. S. Passman, The algebraic structure of group rings. Pure and Applied Mathematics. Wiley-Interscience [John Wiley \& Sons], New York-London-Sydney, 1977.

\section{A. Chebotar}

Department of Mathematical Sciences,

Kent State University,

Kent, OH, USA

e-mail: chebotar@math.Kent.edu

P.-H. LEE

Department of Mathematics,

National Taiwan University, Taipei, Taiwan

e-mail: phlee@math.ntu.edu.tw

and

National Center for Theoretical Sciences, Taipei Office,

Taipei, Taiwan 
E. R. PuczyŁOwski

Institute of Mathematics,

University of Warsaw,

Warsaw, Banacha 2, Poland

e-mail: edmundp@minuw.edu.pl

Received: 14 April 2010 